\title{
Nanoarray-Enhanced Micromechanical Pressure Sensor with Remote Optical Readout
}

\author{
Jeong O. Lee ${ }^{1}$, Trong-Tuong Nguyen ${ }^{2}$, David Sretavan ${ }^{2}$, and Hyuck Choo ${ }^{1}$ \\ 1. Department of Electrical Engineering and Medical Engineering, California Institute of Technology, Pasadena, California USA \\ 2. Department of Ophthalmology, University of California. San Francisco, California USA \\ Email:hchoo@caltech.edu
}

\begin{abstract}
We demonstrate a compact implantable intraocular pressure (IOP) sensor with remote optical readout for glaucoma research and patient management. Using non-invasive white light, we excite the sensor's pressure-sensitive optomechanical cavity and detect the reflected light, whose optical signature changes as a function of IOP. The sensor has provided robust measurements of hydrostatic pressure between $10-60 \mathrm{mmHg}$ with an accuracy of $0.15 \mathrm{mmHg}$. OCIS codes: (280.0280) Remote Sensing and Sensors; (280.4788) Optical Sensing and Sensors
\end{abstract}

\section{Introduction}

An elevated IOP is a major risk factor of glaucoma, which is the second leading cause of blindness [1]. Traditional IOP-detection techniques such as tonometry cannot provide direct, accurate, and continuous IOP measurements [2]. LC-resonator-based IOP-sensing implants have been explored yet are limited by the large sizes and short readout distances [3]. Our compact, robust IOP-sensing implant with remote optical readout capability can greatly improve clinical management of glaucoma and accelerate related drug discoveries.

\section{Figures and tables}

Our device is illustrated in Figure 1. The core of the device, the optomechanical cavity, is formed by a flexible Parylene membrane (diameter: $100 \mu \mathrm{m}$; thickness: $3 \mu \mathrm{m}$ ) and rigid silicon substrate whose surfaces are embedded

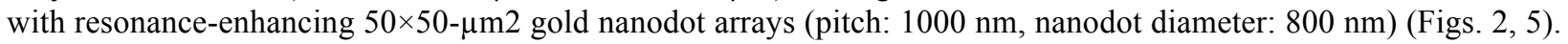
As the pressure is increased from $1 \mathrm{~atm}$, the initial $2-\mu \mathrm{m}$ gap between the Parylene membrane and the silicon (Si) substrate decreases, which shifts the resonant wavelength of the cavity (Fig. 1). To obtain the optimal sensor design parameters that result in highly linear mapping between spectral resonance shifts and pressure changes (1.5 $\mathrm{nm} / \mathrm{mmHg}$ or about three times the typical spectral resolution of a mini-spectrometer), we ran a series of optomechanical FEM and FDTD simulations (using COMSOL and CST), and made experimental measurements.

To fabricate the sensor, we start with photolithography and deep reactive ion etching (DRIE) to create a 2 - $\mu \mathrm{m}$ deep cavity chamber and a recess for adhesive into a Si wafer (Fig. 3a) and patterned a 170-nm-thick gold nanodot array on the Si substrate by performing e-beam lithography and lift-off (Fig. 3b). Then, using DRIE, we created four air holes on the front and on the air reserve chamber on the back of the wafer (Fig. 3c) and patterned a gold nanodot array on the Parylene membrane (Fig. 3d). Finally, using epoxy, we assembled the Parylene membrane and the Si substrate with their nanodot arrays aligned, and sealed the air reserve chamber with a Si chip (Figs. 3e-f).

We tested the device inside a water-filled pressure chamber connected to a pressure gauge and regulator (Fig. 4). Using a broadband source (780-1150 nm for minimum absorption in water and tissue) and a mini-spectrometer (0.22-nm spectral resolution), we remotely acquired reflection spectra from the sensor in the chamber at a 7-mm distance (measurement-setup limited) (Fig. 6). We linearly varied the chamber pressure and tracked the locations of the major peaks in the spectra, which produced a highly linear mapping of resonance shifts to pressure changes (Fig. 7). The average values and standard deviation of the peak locations were determined from over 300 measurements. The sensitivity of the device is $1.53 \mathrm{~nm} / \mathrm{mmHg}$ over the range of $10-60 \mathrm{mmHg}$ with an accuracy of $0.15 \mathrm{mmHg}$. The presence of the nanodot array significantly enhances the signal-to-noise ratio of the sensor by a factor of seven and enables robust remote detection. As a next step, we are pursuing measurements in ex vivo rabbit eyes. Figure 8 shows robust optical resonance captured remotely over a 7-mm distance from an earlier prototype device (consisting of two rigid membranes) implanted in the anterior chamber of an ex vivo rabbit eye. We are also working on smaller devices for use in rodent eyes and the setup with a longer readout distance (theoretical limit $\sim 20 \mathrm{~cm}$ ).

\section{References}

[1] Morrison, J., \& Pollack, I. (2002). Glaucoma: Science and Practice. Thieme.

[2] Katuri, K. C., Asrani, S., \& Ramasubramanian, M. K. (2008). Intraocular pressure monitoring sensors. Sensors Journal, IEEE, 8(1), 12-19.

[3] Chen, P. J., Saati, S., Varma, R., Humayun, M. S., \& Tai, Y. C. (2010). Wireless intraocular pressure sensing using microfabricated minimally invasive flexible-coiled LC sensor implant. J. of Microelectromechanical Systems, 19(4), 721-734. 
a)

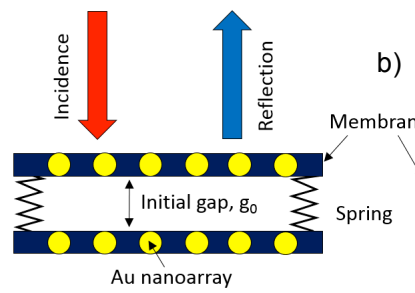

c)

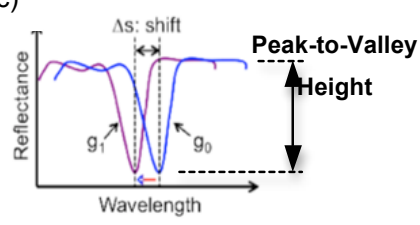

Figure 1. Schematic of IOP sensor: a) Disk-shaped optomechanical cavity. b) Membrane deformation by ambient pressure. c) Resulting shift in resonance due to gap reduction. a)

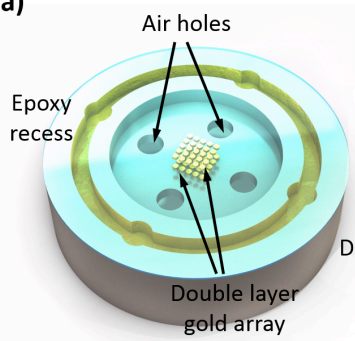

b)

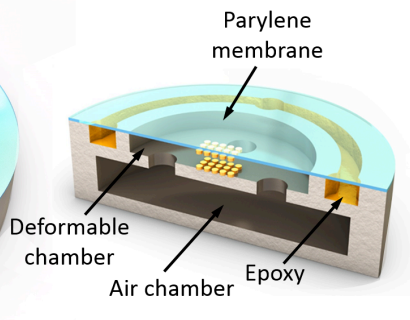

Figure 2. a) 3-D illustration of the sensor. b) Cross-sectional view.

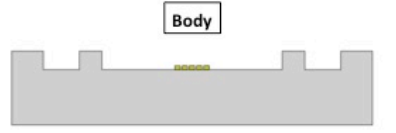

a) Silicon etching by DRIE. (2um)

b) Gold nanodot array fabricated by e-beam lithography and lift-off. (Cr/Au $5 \mathrm{~nm} / 170 \mathrm{~nm})$

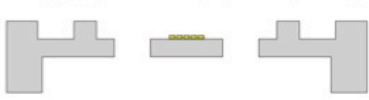

c) Release hole and backside gas chamber etched by backside DRIE.

Si Parylene Au Epoxy

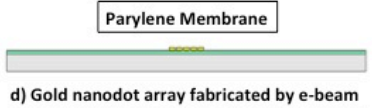

d) Gold nanodot array fabricated by e-beam
lithography and lift-off. (Cr/Au $5 \mathrm{~nm} / 170 \mathrm{~nm}$ ).

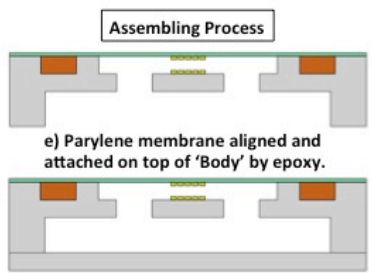

f) Backside gas chamber sealed by another Si chip.
Figure 3. Fabrication process
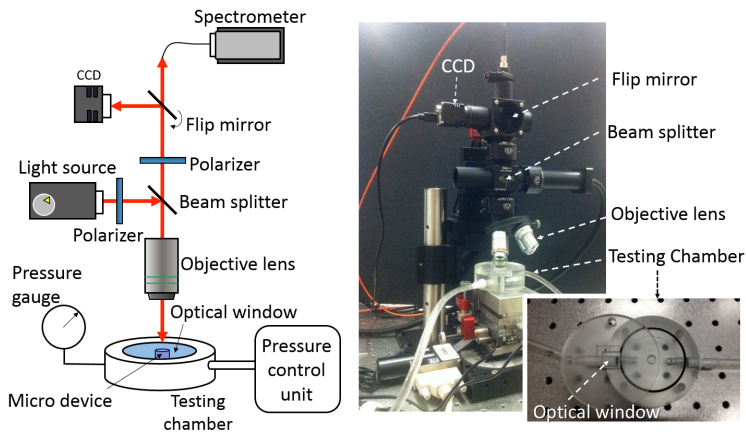

Figure 4: A schematic diagram and a photo of the measurement setup. The inset in the bottom-right corner shows the in-house built pressure chamber that emulates the environment of the anterior chamber.
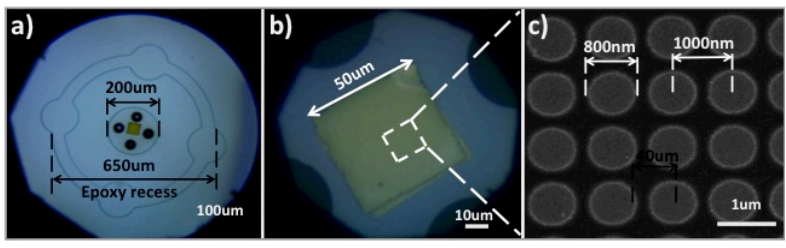

Figure 5. Images of the fabricated device: a) Entire device (top view) b) Two aligned gold nanodot arrays. c) SEM image of a gold nanodot array on Parylene membrane.

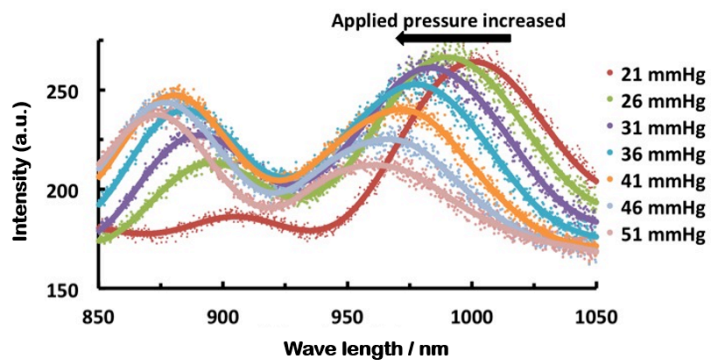

Figure 6. Spectral data points showing resonance shifts in response to pressure changes from $21-51 \mathrm{mmHg}$.

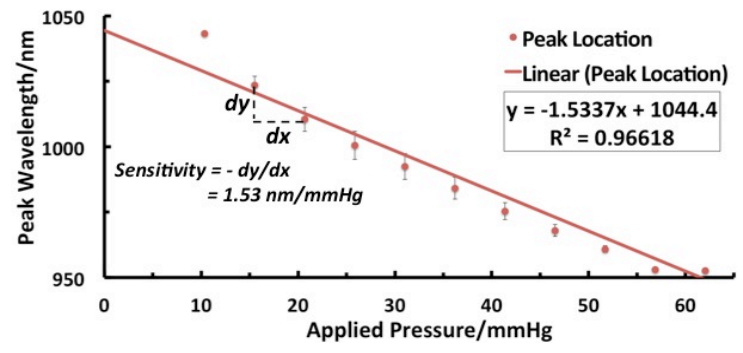

Figure 7: Linear mapping between the shift in peak locations and the ambient hydrostatic pressure: the linearity is 0.96618 .

a)

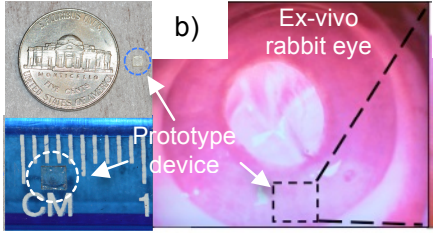

c)

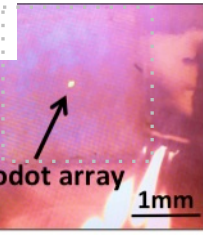

d)

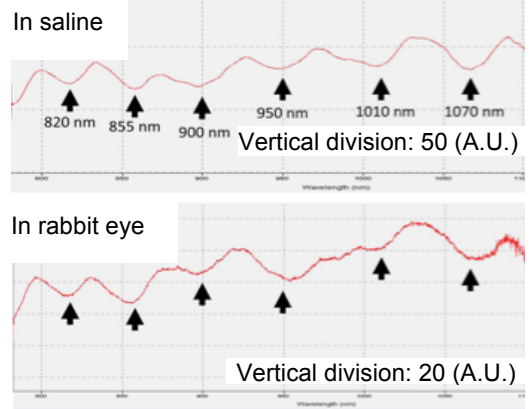

Figure 8 : a) Earlier prototype device, $\sim 2 \mathrm{~mm}$ by $2 \mathrm{~mm}$. b) Prototype implanted in the anterior chamber of an ex-vivo rabbit eye. c) A zoomin image of the implant in the ex-vivo rabbit eye - the gold nanodot array appears as a bright spot in the image, indicated by the arrow. d) A resonance spectrum captured from the prototype device implanted in ex-vivo rabbit eye and also in saline: The resonance dips appear exactly at the identical locations. 